\title{
Diammonium glycyrrhizinate lipid ligand ameliorates lipopolysaccharide-induced acute lung injury by modulating vascular endothelial barrier function
}

\author{
MEI-MEI LIU, JIN ZHOU, DAN JI, JUN YANG, YAN-PING HUANG and QI WANG \\ Department of Histology and Embryology, Anhui Medical College, Hefei, Anhui 230601, P.R. China
}

Received April 26, 2020; Accepted November 24, 2020

DOI: $10.3892 /$ etm.2021.9734

\begin{abstract}
The aim of the present study was to investigate the effects of diammonium glycyrrhizinate lipid ligand (DGLL) treatment on acute lung injury (ALI) and pulmonary edema induced by lipopolysaccharide (LPS) in Sprague-Dawley rats. Rats orally received 30, 60 and $120 \mathrm{mg} / \mathrm{kg}$ DGLL. After $1 \mathrm{~h}$, the rat ALI model was established by LPS $(10 \mathrm{mg} / \mathrm{kg})$ intraperitoneal injection. After $6 \mathrm{~h}$, lung injury was evaluated using hematoxylin and eosin staining techniques. Pulmonary edema was evaluated using lung wet-dry weight ratio, protein concentrations in the bronchoalveolar lavage fluid (BALF) and Evans blue (EB) extravasation in lung tissue. The expression levels of tumor necrosis factor (TNF)- $\alpha$ and interleukin (IL)-1 $\beta$ in lung tissues were measured using ELISA. Myeloperoxidase (MPO) expression levels were detected by immunohistochemical staining. Western blotting was used to measure the expression level changes of intercellular adhesion molecule (ICAM)-1, as well as adherent and tight junction proteins, including vascular endothelial (VE)-cadherin, zonula occludens (ZO)-1, occludin and junctional adhesion molecule (JAM)-1 that were associated with pulmonary inflammation and microvascular permeability. DGLL treatment significantly alleviated ALI induced by LPS, which was demonstrated by reduction of MPO-positive cells and expression levels of TNF- $\alpha$, IL-1 $\beta$ and ICAM-1 in rat lung tissues. In addition, DGLL abrogated LPS-induced pulmonary edema, decreased the protein concentration in BALF and reduced EB extravasation. DGLL also reversed the reduced expression of VE-cadherin and tight
\end{abstract}

Correspondence to: Professor Qi Wang, Department of Histology and Embryology, Anhui Medical College, 632 Fu Rong Road, Hefei, Anhui 230601, P.R. China

E-mail: 41722315@qq.com

Abbreviations: ALI, acute lung injury; ARDS, acute respiratory distress syndrome; BALF, bronchoalveolar lavage fluid; DGLL, diammonium glycyrrhizinate lipid ligand; EB, Evans blue; LPS, lipopolysaccharide; MPO, myeloperoxidase; SD, Sprague-Dawley

Key words: inflammatory infiltration, adhesion molecule, vascular endothelial-cadherin, tight junction protein junction proteins, including ZO-1, occludin and JAM-1 in the lung tissues caused by LPS. In conclusion, DGLL exhibits a protective effect on LPS-induced rat ALI, which is associated with the inhibition of inflammatory cell infiltration and microvascular barrier disruption. The present results provide a theoretical basis for the application of DGLL for the potential clinical treatment of ALI.

\section{Introduction}

Acute lung injury (ALI) is characterized by pulmonary edema and atelectasis as a result of diffuse alveolar-capillary membrane damage, which manifests as respiratory distress and refractory hypoxemia in patients (1). ALI is a common but serious consequence of a number of pathological conditions, including severe infection, trauma, shock and harmful gas inhalation (2). Acute respiratory distress syndrome (ARDS) is a severe form of ALI that can rapidly progress into multiple organ failure, which has a poor prognosis among patients (3). Anti-inflammatory treatments, such as corticosteroids, are currently the primary method for the clinical treatment of ALI (4). However, despite exhibiting significant inhibitory effects on the inflammatory response during ALI, clinical trials have previously demonstrated that hormonal drugs can cause unforeseen adverse side effects in clinical use (5). In addition, they do not reduce the mortality rate of patients with ALI (5). Other inhaled anti-asthmatic drugs, including activated protein $\mathrm{C}$, albuterol and surfactants, have all been withdrawn due to poor clinical efficacy or side effects (6). In recent years, a new understanding on the pathogenesis and pathophysiology of ALI/ARDS has emerged (7,8). Clinical and experimental studies have demonstrated that pulmonary capillary barrier injury followed by increased pulmonary edema is the most important pathological feature of ALI/ARDS, providing a basis for the early stages of this condition $(9,10)$. However, there remains to be a lack of effective therapeutic strategies for treating increased pulmonary microvascular permeability (11).

Diammonium glycyrrhizinate lipid ligand (DGLL) is an extract of the active ingredient of the root of the Chinese licorice Glycyrrhiza uralensis. The primary active component of DGLL is the glycyrrhizic acid diammonium glycyrrhizinate (Fig. 1A), which is a natural major bioactive pentacyclic triterpenoid glycoside that possesses 
comprehensive pharmacological properties, including anti-hepatitis, antiviral, anti-inflammatory, anti-allergy, antioxidant and antitumor characteristics (12-14). This traditional Chinese medicinal licorice has been widely used as an active component in preparations, such as Mahuang and Maxing Shigan decoctions, for treating respiratory infection and acute lung injury $(15,16)$. Modern clinical observations have demonstrated that glycyrrhizin alone or in combination with other agents can effectively ameliorate lung injury, improve alveolar gas exchange and inhibit pulmonary inflammation (17-19). In addition, previous studies have shown that licorice exhibits significant inhibitory effects on inflammatory responses to non-alcoholic fatty liver in rats, by suppressing inflammatory mediator expression and inhibiting leukocyte adhesion, infiltration and peroxide release $(20,21)$.

However, the potential effects of DGLL on pulmonary edema, is role in ALI and underlying mechanism remain to be fully elucidated. Therefore, the present study investigated the effects and underlying mechanisms of DGLL on ALI and pulmonary edema induced by lipopolysaccharide (LPS) in rat models.

\section{Materials and methods}

Animals and drugs. Male Sprague-Dawley (SD) rats (113 in total) weighing 180-200 g (age, $6 \pm 1$ weeks) were provided by Anhui Medical University Experimental Animal Center (Hefei, China). The animals were given tap water ad libitum and housed at $24 \pm 1^{\circ} \mathrm{C}$ and relative humidity of $50 \pm 1 \%$ with a light/dark cycle of $12 \mathrm{~h}$. Animals were fed with standard grain forage and kept at room temperature $\left(18-22^{\circ} \mathrm{C}\right)$ for 1 week with free access to food and water before the experiments and animal health and behavior were monitored once a day. All animals were handled according to the guidelines of the Anhui Medical University Animal Research Committee (22). The protocols were approved by the Committee on the Ethics of Animal Experiments of the Anhui Medical University (approval nos. IACUC20180724-18 and IACUC20200710-11; Hefei, China).

DGLL enteric-coated capsules (cat. no. 20180126AD), also known as Tianqing Ganping, were provided by Jiangsu Zhengda Datianqing Pharmacy Co., Ltd.

Experimental protocol. The present study was divided into two parts. In the first set of experiments, SD rats were randomly divided into the following six groups: i) Sham group $(n=18)$; ii) DGLL group ( $\mathrm{n}=3)$, which received $120 \mathrm{mg} / \mathrm{kg}$ alone; iii) LPS (Escherichia coli serotype O55:B5, Sigma-Aldrich; Merck KGaA) group ( $\mathrm{n}=18)$, which received $10 \mathrm{mg} / \mathrm{kg}$ LPS for $6 \mathrm{~h}$; iv) DGLL $30+$ LPS group $(\mathrm{n}=18)$, which received DGLL $30 \mathrm{mg} / \mathrm{kg}+10 \mathrm{mg} / \mathrm{kg}$ LPS; v) DGLL 60 + LPS group $(\mathrm{n}=18)$, which received DGLL $60 \mathrm{mg} / \mathrm{kg}+10 \mathrm{mg} / \mathrm{kg}$ LPS; and vi) DGLL 120 + LPS group $(\mathrm{n}=18)$, which received DGLL $120 \mathrm{mg} / \mathrm{kg}+10 \mathrm{mg} / \mathrm{kg}$ LPS (Table I).

In the Sham group, physiological saline $(5 \mathrm{ml} / \mathrm{kg})$ was given by oral gavage $1 \mathrm{~h}$ after intraperitoneal saline injection $(5 \mathrm{ml} / \mathrm{kg})$. In the DGLL group, DGLL $(120 \mathrm{mg} / \mathrm{kg})$ dissolved in physiological saline was given by oral gavage $1 \mathrm{~h}$ before intraperitoneal saline injection $(5 \mathrm{ml} / \mathrm{kg})$. In LPS group, physiological saline $(5 \mathrm{ml} / \mathrm{kg})$ was given by oral gavage $1 \mathrm{~h}$ before intraperitoneal LPS $(10 \mathrm{mg} / \mathrm{kg})$ injection. In the three DGLL pre-treatment groups, DGLL (30, 60 and $120 \mathrm{mg} / \mathrm{kg}$ ) dissolved in physiological saline was administered by oral gavage $1 \mathrm{~h}$ before intraperitoneal LPS $(10 \mathrm{mg} / \mathrm{kg})$ injection. In total, $6 \mathrm{~h}$ after LPS injection, rats were anesthetized with $2 \%$ pentobarbital $(60 \mathrm{mg} / \mathrm{kg})$ by intraperitoneal injection, where the successful induction of anesthesia was defined as immobility and the absence of motor response of rats to a noxious stimulus (such as a pinch). The rats were euthanized by exsanguination followed by cardiac arrest under anesthesia at the indicated endpoint of experiment.

In the second set of experiments, rats were randomly divided into the following five groups: i) Sham group; ii) LPS group, which received $10 \mathrm{mg} / \mathrm{kg}$ LPS alone for $1 \mathrm{~h}$; iii) DGLL $30+$ LPS group, which received $30 \mathrm{mg} / \mathrm{kg}$ DGLL and + $10 \mathrm{mg} / \mathrm{kg}$ LPS for $1 \mathrm{~h}$; iv) DGLL $60+$ LPS group, which received $60 \mathrm{mg} / \mathrm{kg}$ DGLL and $10 \mathrm{mg} / \mathrm{kg}$ LPS for $1 \mathrm{~h}$; and v) DGLL 120 + LPS group, which received $120 \mathrm{mg} / \mathrm{kg}$ DGLL and $10 \mathrm{mg} / \mathrm{kg}$ LPS for $1 \mathrm{~h}$ (Table II). The animal treatment protocol was the same as that for that described for the LPS $6 \mathrm{~h}$ groups aforementioned. A total of $1 \mathrm{~h}$ after LPS injection, rats were sacrificed for parameter detection.

Cell culture. RAW 264.7 murine macrophages were obtained from ScienCell Research Laboratories, Inc. The cells were cultured at a density of $1 \times 10^{5}$ cells $/ \mathrm{cm}^{2}$ in DMEM supplemented with $10 \%$ of FBS (both from ScienCell Research Laboratories, Inc.), streptomycin $(100 \mu \mathrm{g} / \mathrm{ml})$ and penicillin $(100 \mathrm{U} / \mathrm{ml})$. The cells were cultured to confluence under a humidified atmosphere of $5 \% \mathrm{CO}_{2}$ and $95 \%$ air at $37^{\circ} \mathrm{C}$ and incubated with LPS $(100 \mathrm{ng} / \mathrm{ml})$ for $6 \mathrm{~h}$ at $37^{\circ} \mathrm{C}$. In the DGLL pre-treatment groups, DGLL was added $1 \mathrm{~h}$ before LPS stimulation to a concentration of 50,100 and $200 \mu \mathrm{g} / \mathrm{ml}$.

Hematoxylin and eosin $(H \& E)$ staining. At $6 \mathrm{~h}$ after LPS stimulation, rats were anesthetized and euthanatized before the middle right lobe of the lung was cut from each rat for fixation in $4 \%$ paraformaldehyde for $48 \mathrm{~h}$ at $4{ }^{\circ} \mathrm{C}$ and processed for paraffin sectioning $(5 \mu \mathrm{m})$. H\&E staining was performed to evaluate lung tissue injury. In brief, after deparaffinization by xylene and ethanol gradient, sections were stained by sequential hematoxylin for $5 \mathrm{~min}$ and eosin for $15 \mathrm{sec}$, both at room temperature. Using a light microscope (Olympus, Tokyo, Japan), the morphological changes of lung tissue were observed under a $\times 10$ objective lens. Five visual fields were selected randomly from each section, and lung injury score was determined based on the following histological features: i) Focal alveolar membrane thickening; ii) capillary congestion; iii) intra-alveolar hemorrhage; iv) interstitial edema; and v) intra-alveolar leukocyte infiltration. Each feature was scored from 0 to 3 based on its presence: i) Score 0, 0\% of the section areas; ii) mild, score $1,0-25 \%$ of the section areas; iii) moderate, score $2,25-50 \%$ of the section areas; and iv) severe, score $3>50 \%$ of the section areas. The total lung injury score was the sum of the score of each feature (15 represents the maximum and the most severe) (23).

Myeloperoxidase (MPO) immunohistochemical staining. MPO expression levels in the lung tissues were determined by immunohistochemistry. Briefly, sections were deparaffinized 
Table I. Allocation of animals into the different experimental groups and parameters measured at $6 \mathrm{~h}$ after LPS injection.

\begin{tabular}{|c|c|c|c|c|c|c|c|c|}
\hline Experimental group & $\begin{array}{l}\text { Wet-to-dry } \\
\text { ratio }\end{array}$ & ELISA & $\begin{array}{l}\text { Western } \\
\text { blotting }\end{array}$ & $\mathrm{H} \& \mathrm{E}$ & IHC and IF & $\begin{array}{c}\text { Evans blue } \\
\text { extravasation }\end{array}$ & $\begin{array}{c}\text { BALF } \\
\text { analysis }\end{array}$ & Total \\
\hline Sham & 6 & $6^{\mathrm{a}}$ & $4^{\mathrm{a}}$ & $3^{\mathrm{a}}$ & $3^{\mathrm{a}}$ & 6 & 6 & 18 \\
\hline LPS & 6 & $6^{\mathrm{a}}$ & $4^{\mathrm{a}}$ & $3^{\mathrm{a}}$ & $3^{\mathrm{a}}$ & 6 & 6 & 18 \\
\hline DGLL 30 + LPS & 6 & $6^{\mathrm{a}}$ & $4^{\mathrm{a}}$ & $3^{\mathrm{a}}$ & $3^{\mathrm{a}}$ & 6 & 6 & 18 \\
\hline DGLL 60 + LPS & 6 & $6^{\mathrm{a}}$ & $4^{\mathrm{a}}$ & $3^{\mathrm{a}}$ & $3^{\mathrm{a}}$ & 6 & 6 & 18 \\
\hline DGLL 120 + LPS & 6 & $6^{\mathrm{a}}$ & $4^{\mathrm{a}}$ & $3^{\mathrm{a}}$ & $3^{\mathrm{a}}$ & 6 & 6 & 18 \\
\hline DGLL & & & & 3 & $3^{\mathrm{b}}$ & & & 3 \\
\hline Total & 30 & & & 3 & & 30 & 30 & 93 \\
\hline
\end{tabular}

${ }^{\text {aS }}$ Same group of rats as that used for wet-to-dry weight ratio; 'bame group of rats were used as that used for H\&E. H\&E, hematoxylin and eosin; LPS, lipopolysaccharide; DGLL, diammonium glycyrrhizinate lipid ligand; BALF, bronchoalveolar lavage fluid; IHC, immunohistochemistry; IF, immunofluorescence.

Table II. Number of animals allocated into each of the different experimental groups for analysis $1 \mathrm{~h}$ after LPS injection.

\begin{tabular}{lcr}
\hline $\begin{array}{l}\text { Experimental } \\
\text { group }\end{array}$ & $\begin{array}{c}\text { Western blotting analysis } \\
\text { for phosphorylated-VE cadherin }\end{array}$ & Total \\
\hline Sham & 4 & 4 \\
LPS & 4 & 4 \\
DGLL 30 + LPS & 4 & 4 \\
DGLL 60 + LPS & 4 & 4 \\
DGLL 120 + LPS & 4 & 4 \\
Total & 20 & 20 \\
\hline
\end{tabular}

LPS, lipopolysaccharide; DGLL, diammonium glycyrrhizinate lipid ligand; VE cadherin, vascular endothelial cadherin.

using xylene and ethanol gradient followed by heat-mediated antigen retrieval in $0.01 \mathrm{M}$ citrate buffer ( $\mathrm{pH}$ 6.0) and hydrogen peroxide $(0.3 \%)$ blocking at room temperature for $30 \mathrm{~min}$. Then, sections were blocked with goat serum (cat. no. ZLI-9056; OriGene Technologies, Inc.) for $30 \mathrm{~min}$ at room temperature and incubated overnight with the rabbit MPO polyclonal primary antibody (1:200; Abcam; cat. no. ab9535) at $4^{\circ} \mathrm{C}$. The sections were then incubated with biotinylated anti-rabbit IgG-horseradish peroxidase (HRP) (cat. no. SP-9001, OriGene Technologies, Inc.) at room temperature for $30 \mathrm{~min}$ and visualized using a DAB substrate kit (OriGene Technologies, Inc.). The location and expression levels of MPO-positive cells in lung tissue samples were observed under a light microscope under a x20 objective lens. A total of five visual fields were selected from each section for analysis of the numbers of positive cells.

Occludin immunofluorescence staining. Tissue sections were deparaffinized by xylene and ethanol gradient followed by heat-mediated antigen retrieval in $0.01 \mathrm{M}$ citrate buffer (pH 6.0), washed with PBS and permeabilized with $0.3 \%$ Triton X-100 for $30 \mathrm{~min}$ at $37^{\circ} \mathrm{C}$. Following blocking with $3 \%$ goat serum (cat. no. ZLI-9056; OriGene Technologies, Inc.) at room temperature for $30 \mathrm{~min}$, sections were incubated with primary antibodies against Occludin (1:50, Invitrogen; Thermo Fisher Scientific, Inc.; cat. no. 33-1500) and von Willebrand factor (1:50; Abcam; cat. no. ab6994) diluted in PBS overnight at $4^{\circ} \mathrm{C}$. After being rinsed with PBS, sections were incubated with Dylight ${ }^{\mathrm{TM}}$ 488-labeled goat-anti rabbit secondary antibodies (1:100; KPL, Inc.; cat. no. 5230-0385) and Dylight ${ }^{\mathrm{TM}}$ 549-labeled goat-anti mouse secondary antibodies (1:100; KPL, Inc.; cat. no. 072-04-18-03) for $2 \mathrm{~h}$ at $37^{\circ} \mathrm{C}$. All sections were counterstained with Hoechst 33342 (1:50; Dojindo Molecular Technologies, Inc.) for $20 \mathrm{~min}$ at room temperature to stain the nuclei. Images were acquired using a laser scanning confocal microscope under a x63 objective lens (Leica Microsystems $\mathrm{GmbH}$ ).

ELISA. At $6 \mathrm{~h}$ after LPS stimulation, rats were anesthetized and the right middle lobe of the lung from each rat was collected. The one-step RIPA (Applygene Technologies, Inc.) method was used to extract total protein from lung tissues. The expression levels of tumor necrosis factor- $\alpha$ (TNF- $\alpha$; cat. no. RTA00) and interleukin (IL)-1 $\beta$ (cat. no. RLB00) in the lung tissues and cell culture supernatants of RAW 264.7 cells were measured using an ELISA kit (R\&D Systems, Inc.) according to the manufacturer's protocols.

Determination of the lung dry-wet weight ratio. At $6 \mathrm{~h}$ after LPS stimulation, rats were anesthetized and $100 \mathrm{mg}$ lung tissue from each rat was sampled from the right upper lobe. The lung tissue was then weighed before the tissue was dried in a vacuum oven at $80^{\circ} \mathrm{C}$ for $48 \mathrm{~h}$ and the lung tissue was weighed again. The ratio of the lung weight before and after oven drying was then calculated.

Pulmonary microvascular permeability test. At $6 \mathrm{~h}$ after the intraperitoneal injection of LPS, rats were anesthetized with $2 \%$ pentobarbital $(60 \mathrm{mg} / \mathrm{kg})$ by peritoneal injection, before being injected with $2 \%$ Evans blue (EB) solution $(30 \mathrm{mg} / \mathrm{kg})$ via the jugular vein. After $30 \mathrm{~min}$, rats were sacrificed by perfusion with saline via the right ventricle until cardiac arrest to rinse intravascular EB in the lung tissue and part of the lower left lobe lung tissue of each rat was collected, weighed 
and placed in a clean centrifuge tube. A total of $\sim 100 \mathrm{mg}$ wet weight of the lower left lobe lung tissue was added to $1 \mathrm{ml}$ $100 \%$ formamide, placed in a water bath at $37^{\circ} \mathrm{C}$ for $24 \mathrm{~h}$ and centrifuged at $1,000 \mathrm{xg}$ at room temperature for $30 \mathrm{~min}$ before the supernatant was extracted. Absorbance values of $200 \mu \mathrm{l}$ supernatant were then measured using a spectrophotometer at the wavelength of $620 \mathrm{~nm}$. The standard curve method was used to calculate the EB content of each sample. Pulmonary microvascular permeability was expressed as the ratio of EB content to the wet lung weight.

Bronchoalveolar lavage fluid (BALF) collection and analysis. At $6 \mathrm{~h}$ after intraperitoneal injection of LPS, rats were anesthetized before a plastic cannula was inserted into the trachea to collect BALF by sterile physiological saline aspiration, which was performed three times. The rats were then euthanized by exsanguination until cardiac arrest. The BALF sample was centrifuged at $1,300 \mathrm{xg}$ at $4^{\circ} \mathrm{C}$ for $10 \mathrm{~min}$ and the supernatant was extracted. Bicinchoninic acid protein assay (BCA) method was used to detect total protein concentration in the BALF samples, whilst the cell pellet was resuspended in PBS. The cells were then stained with Wright-Giemsa stain for $1 \mathrm{~min}$ at room temperature and the total cell counts were assessed using a cell count chamber and observed under a light microscope under a $\mathrm{x} 4$ objective lens.

Western blot analysis. At $6 \mathrm{~h}$ after LPS stimulation, rats were anesthetized and euthanatized, and the right lower lobe of each rat was sampled. The one-step RIPA (Applygene Technologies, Inc.) method was used to extract total protein from lung tissues and RAW 264.7 macrophages before protein concentration was measured using the BCA method. Following separation by $8 \%$ SDS-PAGE electrophoresis (100 $\mathrm{mg}$ protein for each group), the protein samples were transferred onto PVDF membranes for blocking by $5 \%$ non-fat milk at room temperature for $1 \mathrm{~h}$. The following primary antibodies and concentrations were used: intercellular adhesion molecule (ICAM)-1 (1:1,000; Abcam; cat. no. ab206398), vascular endothelial (VE)-cadherin (1:500; Invitrogen; Thermo Fisher Scientific, Inc.; cat. no. 36-1900), zonula occludens (ZO)-1 (1:1,000; Invitrogen; Thermo Fisher Scientific, Inc.; cat. no. 40-2200), Occludin (1:500; Invitrogen; Thermo Fisher Scientific, Inc.; cat. no. 33-1500), junctional adhesion molecule (JAM)-1 (1:1,000; Santa Cruz Biotechnology, Inc.; cat. no. sc-53623) and GAPDH (1:5,000; Cell Signaling Technology, Inc.; cat. no. 2118). All primary antibodies were incubated overnight at $4^{\circ} \mathrm{C}$. In a separate experiment, rats were anesthetized $1 \mathrm{~h}$ after LPS stimulation before the right lower lobe was collected and the phosphorylation (p-) of VE-Cadherin (1:1,000; Abcam; cat. no. ab119785) and total VE-cadherin (1:500; Invitrogen; Thermo Fisher Scientific, Inc.; cat. no. 36-1900) were detected. Following incubation with HRP-conjugated anti-rabbit IgG secondary antibody (1:5,000; Cell Signaling Technology, Inc.; cat. no. 7074) or HRP-conjugated anti-mouse IgG secondary antibody (1:5,000; Cell Signaling Technology, Inc.; cat. no. 7076) for $1 \mathrm{~h}$ at room temperature, the bands were visualized using the SuperEnhanced chemiluminescence detection reagents (Applygene Technologies, Inc.). GAPDH was used as the internal reference. The average optical density of western blot bands was measured using Quantity One image analyzer software (v4.6.6; Bio-Rad Laboratories, Inc.) where the ratio of the average optical density of the target band to that of GAPDH was calculated. The results are expressed as the ratio with respect to those of the Sham group.

Statistical analysis. All data are expressed as the mean \pm SEM, $\mathrm{n}=3$ for RAW264.7 cell experiments; $\mathrm{n}=3-6$ for corresponding animal experiments as indicate in Tables I and II. One-way ANOVA and Tukey's post hoc test or Kruskal-Wallis test followed by Dunn's test were performed using SPSS 15.0 mathematical statistics software (SPSS, Inc.). $\mathrm{P}<0.05$ was considered to indicate a statistically significant difference.

\section{Results}

DGLL treatment inhibits LPS-induced ALI and pulmonary inflammation in rats. H\&E staining results showed that in the Sham and DGLL-alone groups, the rat lung structure exhibited completely clear alveolar spaces, where there was no edema or inflammatory cell infiltration in the alveolar septa. By contrast, in the LPS group, notable edema and thickening (Fig. 1B, arrow) occurred in the pulmonary interstitial tissue, which are accompanied by alveolar atrophy. DGLL pre-treatment prevented LPS-induced ALI and insult to the alveolar structure, which were significant at $120 \mathrm{mg} / \mathrm{kg}$ (Fig. 1B and C).

Subsequent immunohistochemistry examination showed that compared with that in the Sham and DGLL-alone groups, MPO-positive cell staining was significantly increased in rat pulmonary tissues following LPS stimulation. MPO-positive staining in the DGLL pretreatment groups was weaker compared with that in the LPS group, where the number of MPO-positive cells appeared to have decreased in a dose-dependent manner (Fig. 1D and E). This suggests that DGLL prevented LPS-induced leukocyte infiltration and damage to the rat pulmonary tissue.

DGLL inhibits LPS-induced expression of cytokines and adhesion molecules in rat lung tissues and macrophages. ELISA data showed that LPS significantly increased inflammatory cytokine TNF- $\alpha$ and IL- $1 \beta$ levels in the lung tissue compared with those in the Sham group (Fig. 2A and B). Pretreatment with 60 and $120 \mathrm{mg} / \mathrm{kg}$ DGLL significantly prevented this increase in LPS-induced inflammatory factor expression in the lung tissue, whilst pretreatment with $30 \mathrm{mg} / \mathrm{kg}$ DGLL exhibited no notable effects (Fig. 2A and B). Similarly, western blot analysis demonstrated that endothelial adhesion molecule ICAM-1 expression levels in the LPS group were significantly increased compared with those in the Sham group. However, pretreatment with 60 and $120 \mathrm{mg} / \mathrm{kg}$ DGLL significantly prevented the LPS-induced upregulation of ICAM-1 levels in the rat lung tissues (Fig. 2C). To validate the anti-inflammatory effects of DGLL, the levels of TNF- $\alpha$ and IL-1 $\beta$ in the cell culture supernatants of RAW 264.7 murine macrophages were next detected. In parallel with the in vivo data, DGLL pretreatment significantly abrogated LPS-induced inflammatory cytokine release, in addition to ICAM-1 expression, by RAW 264.7 cells, at 100 and $200 \mu \mathrm{g} / \mathrm{ml}$ (Fig. 2D-F).

DGLL prevents increases in LPS-induced pulmonary microvascular permeability and pulmonary edema. Compared 


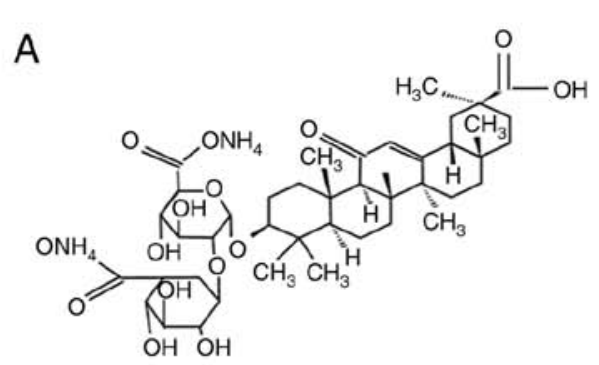

Structure of diammonium glycyrrhizinate

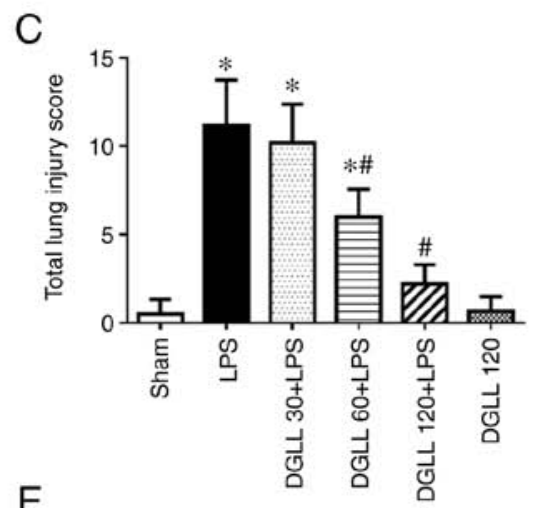

E

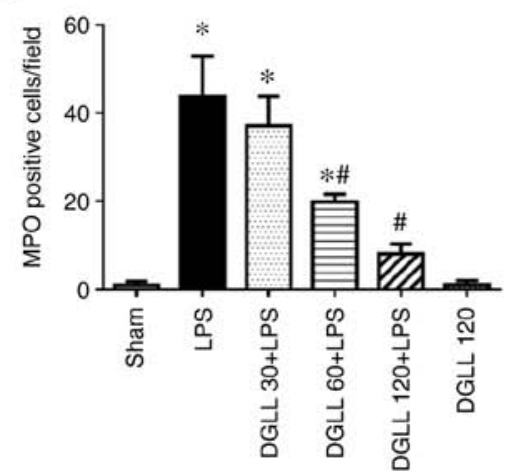

B
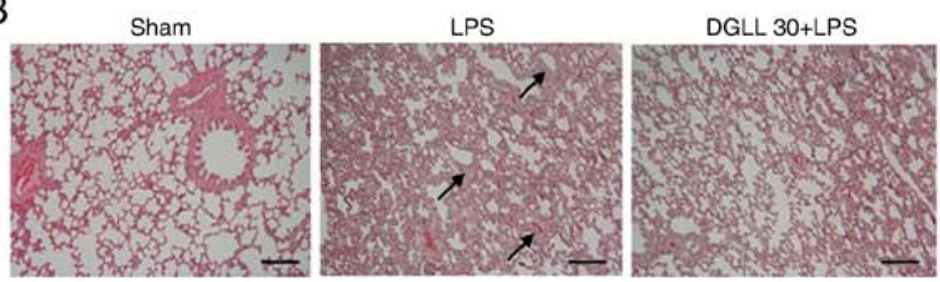

DGLL 60+LPS

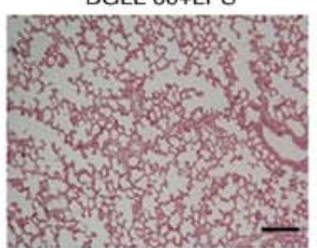

DGLL 120+LPS

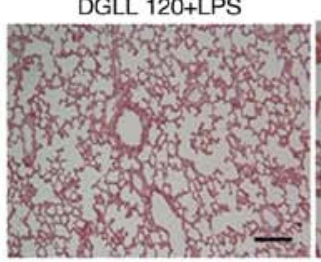

DGLL

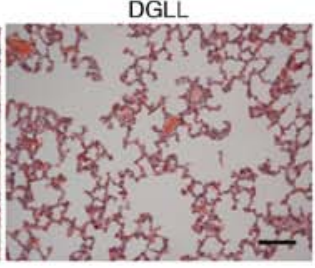

D

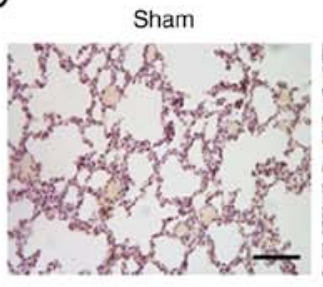

LPS

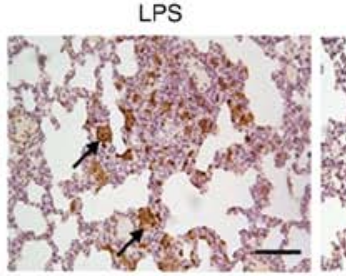

DGLL 30+LPS

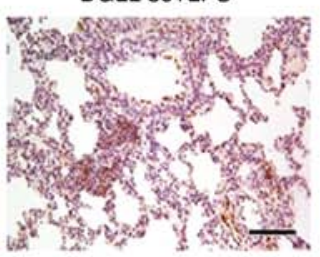

DGLL $60+$ LPS

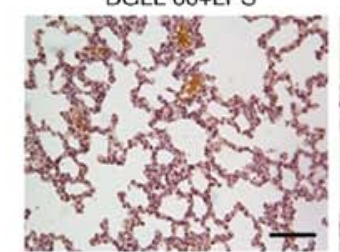

DGLL 120+LPS

DGLL

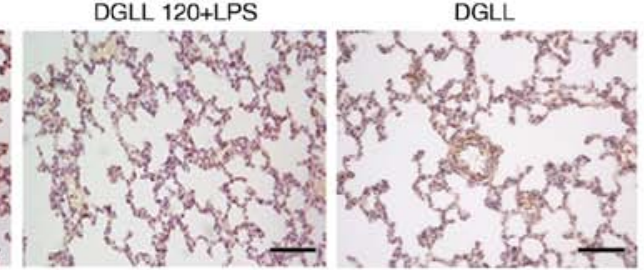

Figure 1. Effect of DGLL on acute lung tissue injury and MPO expression levels in lung tissue following LPS stimulation. (A) Structure of diammonium glycyrrhizinate. (B) Representative H\&E staining images of lung tissue in Sham, LPS and DGLL 30, 60 and $120+$ LPS and DGLL-alone groups. Scale bar, $200 \mu$ m; arrows represent, interstitial edema and thickening. (C) Quantified lung injury score. (D) Representative MPO immunohistochemical staining images of rat lung tissues in Sham, LPS and DGLL 30, 60 and 120 + LPS and DGLL-alone groups. Scale bar, $200 \mu$ m; arrows indicate, MPO-positive cells. (E) Statistical analysis of MPO-positive cells in lung tissues from each condition. All data are expressed as the mean \pm SEM. "P<0.05 vs. Sham; ${ }^{\text {P }<0.05 ~ v s . ~ L P S . ~ D G L L, ~ d i a m m o n i u m ~}$ glycyrrhizinate lipid ligand; MPO, myeloperoxidase; LPS, lipopolysaccharide.

with that in the Sham group, lung tissue edema was significantly increased following LPS induction for $6 \mathrm{~h}$, which was demonstrated by the significant elevation in the wet-to-dry weight ratio of rat lung tissues (Fig. 3A). Extravasation of EB in the lung tissue and the protein content in BALF in the LPS group were also found to be significantly higher compared with those in the Sham group (Fig. 3B and C), suggesting that LPS impaired the integrity of the pulmonary vascular and alveolar epithelial barrier. In addition, a significant increase in the total cell number in BALF was observed following LPS stimulation compared with that in the Sham group (Fig. 3D). All of the aforementioned changes, namely the lung wet/dry weight ratio, EB extravasation, BALF protein concentration and total cell number in BALF, were all significantly lower in the DGLL 60 and $120 \mathrm{mg} / \mathrm{kg}$ groups compared with those in the LPS group (Fig. 3D). These data indicate that pretreatment with DGLL effectively inhibited LPS-induced pulmonary edema and increases in pulmonary microvascular permeability (Fig. 3).
DGLL prevents LPS-induced VE-cadherin phosphorylation and reduction in VE-cadherin expression in rat lung tissues. At $6 \mathrm{~h}$ after LPS induction, western blot analysis showed that the expression levels of the adhesion junction protein VE-Cadherin were significantly reduced in the LPS group, which were significantly prevented by pretreatment with 60 and $120 \mathrm{mg} / \mathrm{kg}$ DGLL (Fig. 4C and D). LPS mediates disruption of VE-Cadherin through a series of transcellular events, starting with the tyrosine phosphorylation of VE-Cadherin (24). Therefore, $p$-VE-cadherin was next measured in lung tissues $1 \mathrm{~h}$ after LPS stimulation. The results showed that levels of VE-cadherin phosphorylation were significantly higher in the LPS group compared with those in the Sham group, which were significantly abrogated by 60 and $120 \mathrm{mg} / \mathrm{kg}$ DGLL pretreatment (Fig. 4A and B). This suggests that DGLL prevented the reductions in VE-cadherin expression by inhibiting LPS-induced VE-cadherin phosphorylation. 

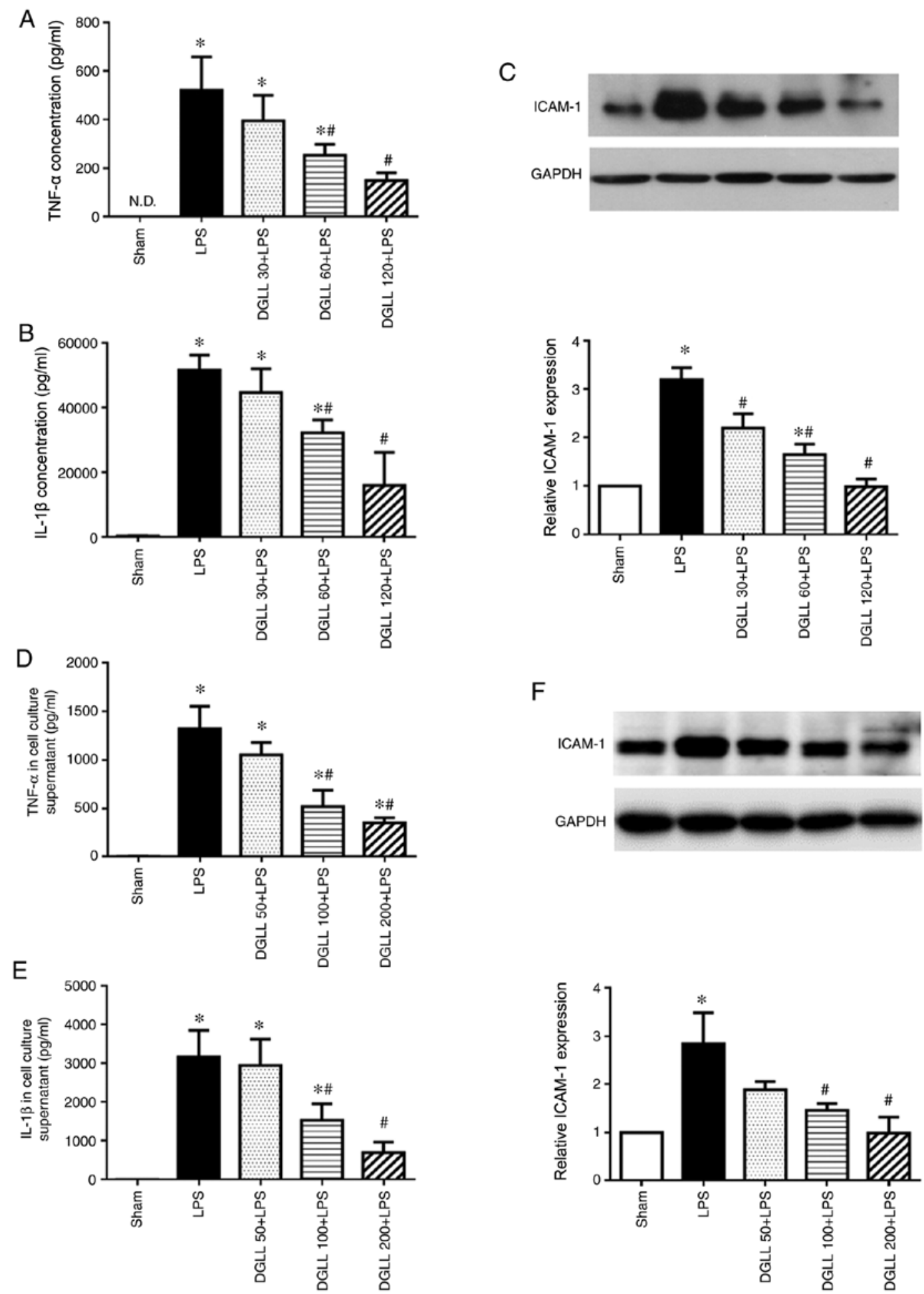

Figure 2. Effect of DGLL on the expression of inflammatory cytokines and adhesion molecules induced by LPS. Expression levels of (A) TNF- $\alpha$ and (B) IL-1 $\beta$ in rat lung tissue as detected by ELISA. (C) Representative western blotting image and corresponding quantification analysis of ICAM-1 expression levels in rat lung tissue. Quantification of the levels of (D) TNF- $\alpha$ and (E) IL-1 $\beta$ in RAW264.7 cell culture supernatant after LPS with/without DGLL treatment as detected by ELISA. (F) Representative western blotting image and corresponding quantification analysis of ICAM-1 expression in RAW264.7 cells. All data are expressed as the mean \pm SEM. "P<0.05 vs. Sham; " $\mathrm{P}<0.05$ vs. LPS. DGLL, diammonium glycyrrhizinate lipid ligand; IL, interleukin; LPS, lipopolysaccharide; ICAM-1, intercellular adhesion molecule-1; TNF, tumor necrosis factor.

DGLL inhibits LPS-induced downregulation of tight junction proteins in rat lung tissues. The role of DGLL in the expression of the tight junction protein occludin was assessed by confocal microscopy at $6 \mathrm{~h}$ after LPS administration (Fig. 5A). Compared with those in the Sham group, reduced occluding expression levels and discontinuous localization of occludin were observed in the rat lung tissues from the LPS group (Fig. 5A-b). These aforementioned effects were markedly prevented by pretreatment with DGLL, particularly at the 60 and $120 \mathrm{mg} / \mathrm{kg}$ (Fig. 5A-d and A-e). Similarly, western blotting results also demonstrated that the expression levels of tight junction proteins $\mathrm{ZO}-1$, occludin 


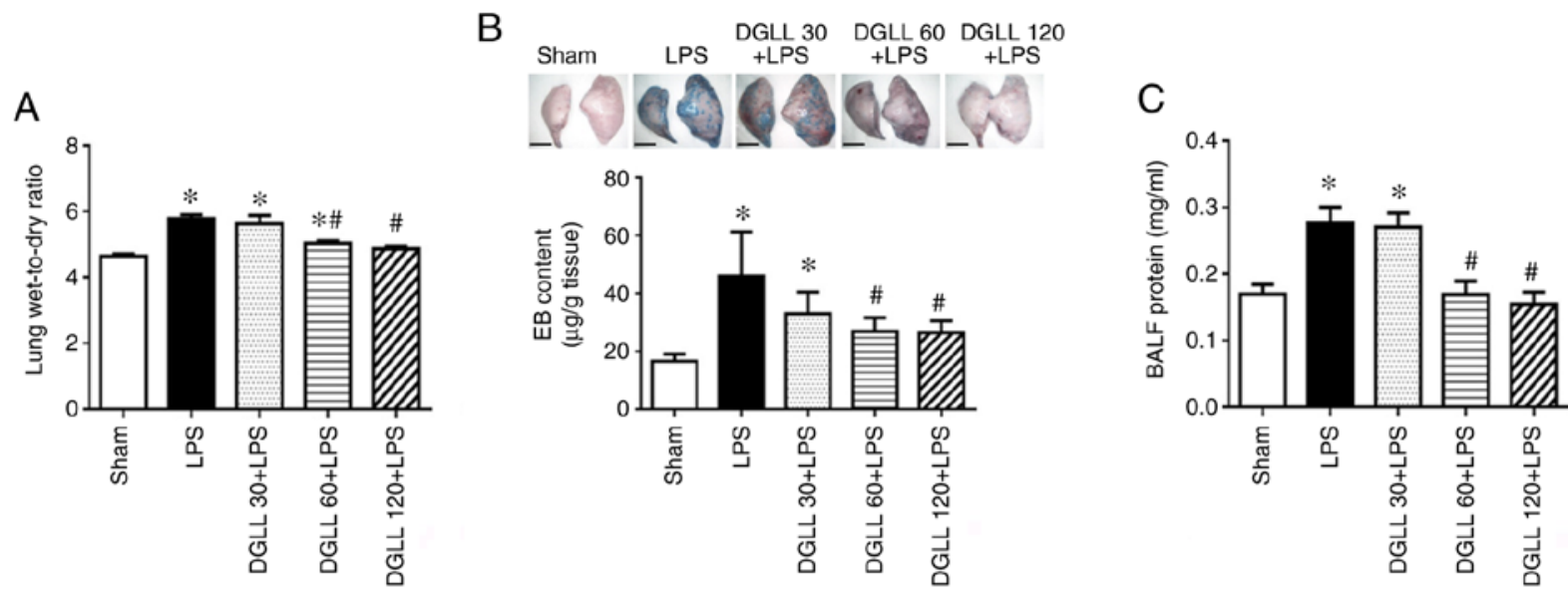

D
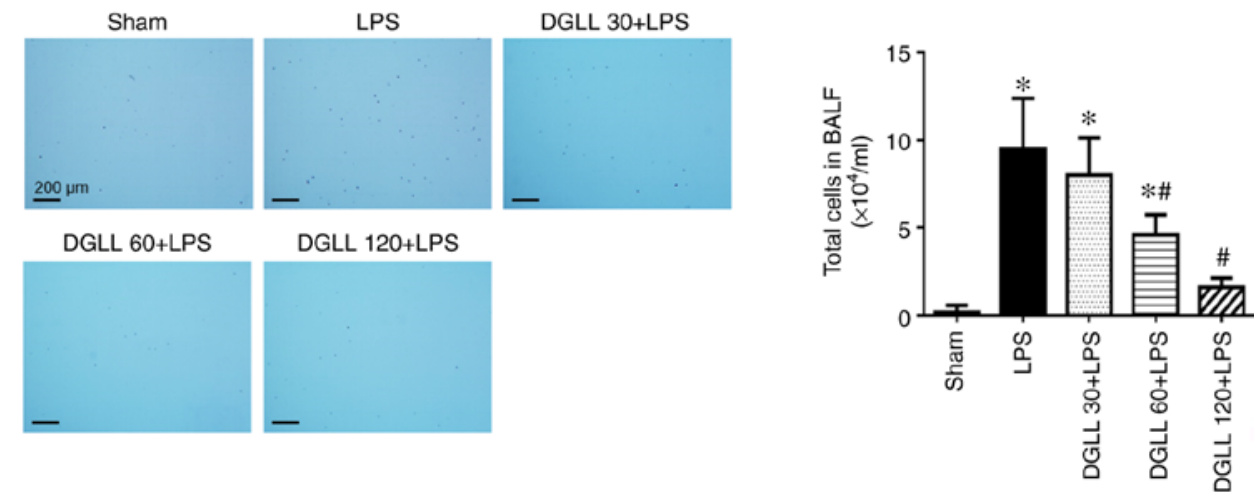

Figure 3. Effect of DGLL on pulmonary microvascular hyperpermeability and pulmonary tissue edema induced by LPS. (A) Wet-to-dry lung weight ratios. (B) Tissue Evans blue content. Scale bar, $500 \mathrm{~mm}$. (C) Protein concentration in BALF. (D) Total cell numbers counted in BALF. All data are expressed as the mean \pm SEM. ${ }^{*} \mathrm{P}<0.05$ vs. Sham; ${ }^{*} \mathrm{P}<0.05$ vs. LPS. LPS, lipopolysaccharide; GLL, diammonium glycyrrhizinate lipid ligand; EB, Evans blue; BALF, bronchoalveolar lavage fluid.

A
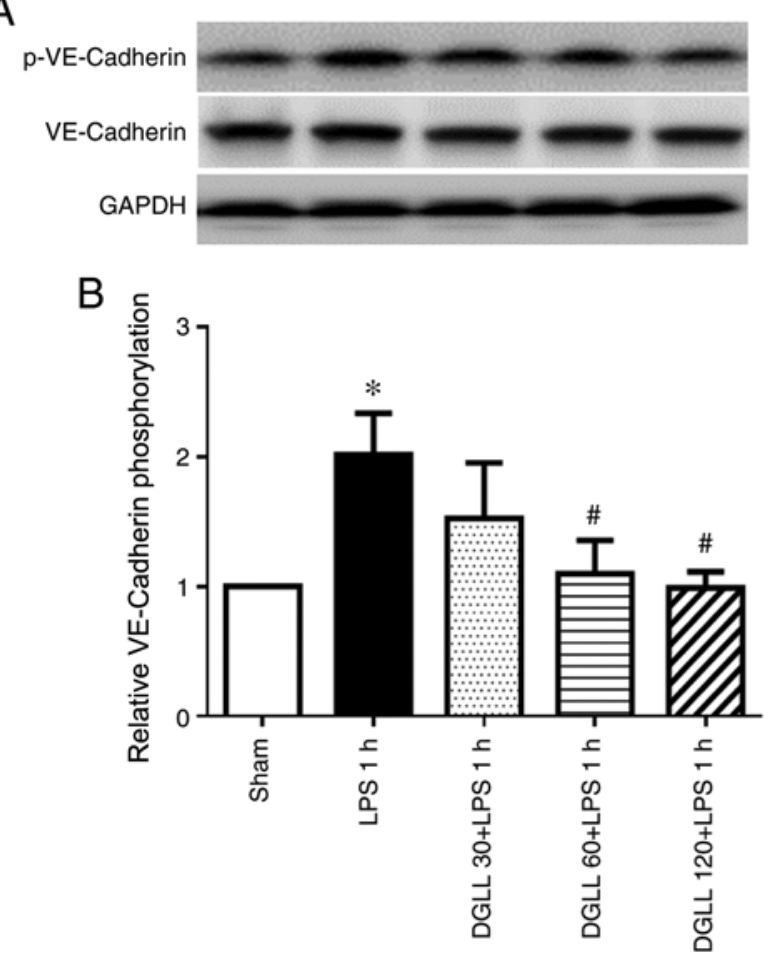

C

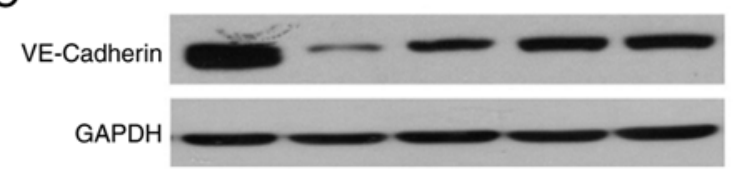

D

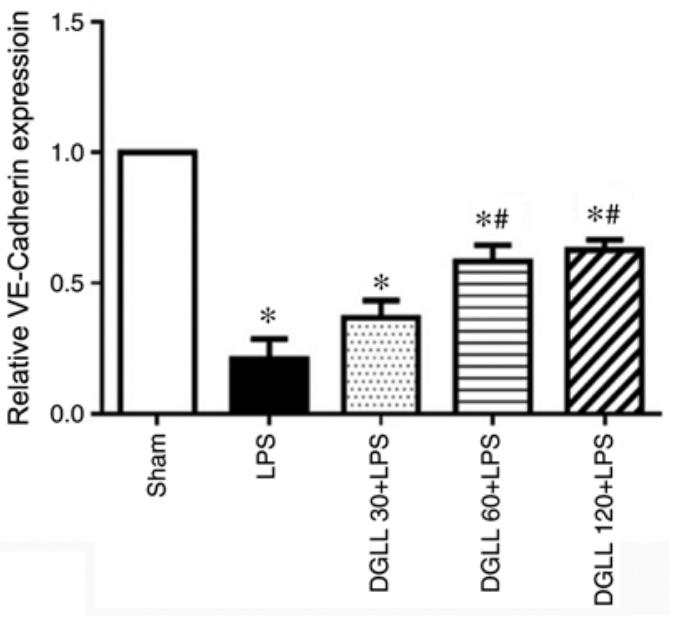

Figure 4. Effect of DGLL on phosphorylation and expression of the adhesion junction protein VE-Cadherin caused by LPS in rat lung tissues. (A) Representative western blotting image and (B) corresponding quantitative analysis of phosphorylated-VE-Cadherin and VE-Cadherin in rat lung tissues $1 \mathrm{~h}$ after LPS stimulation. (C) Representative western blotting image and (D) corresponding quantitative analysis of VE-Cadherin in rat lung tissue $6 \mathrm{~h}$ after LPS stimulation. All data are expressed as the mean \pm SEM. ${ }^{*} \mathrm{P}<0.05$ vs. Sham; ${ }^{\#} \mathrm{P}<0.05$ vs. LPS. DGLL, diammonium glycyrrhizinate lipid ligand; VE, vascular endothelial; LPS, lipopolysaccharide. 
A

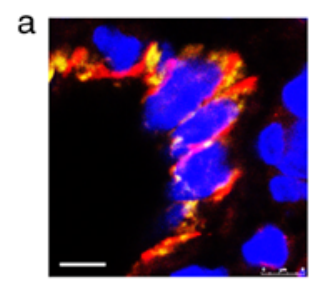

Occludin vWF Nuclear

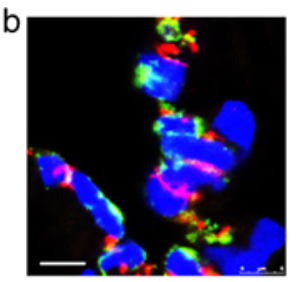

$\mathrm{B}$

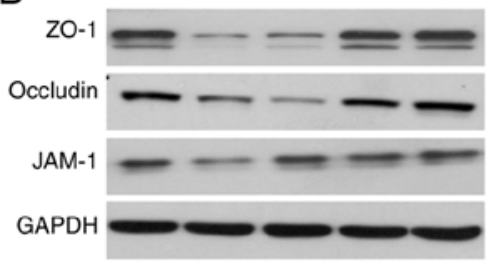

C
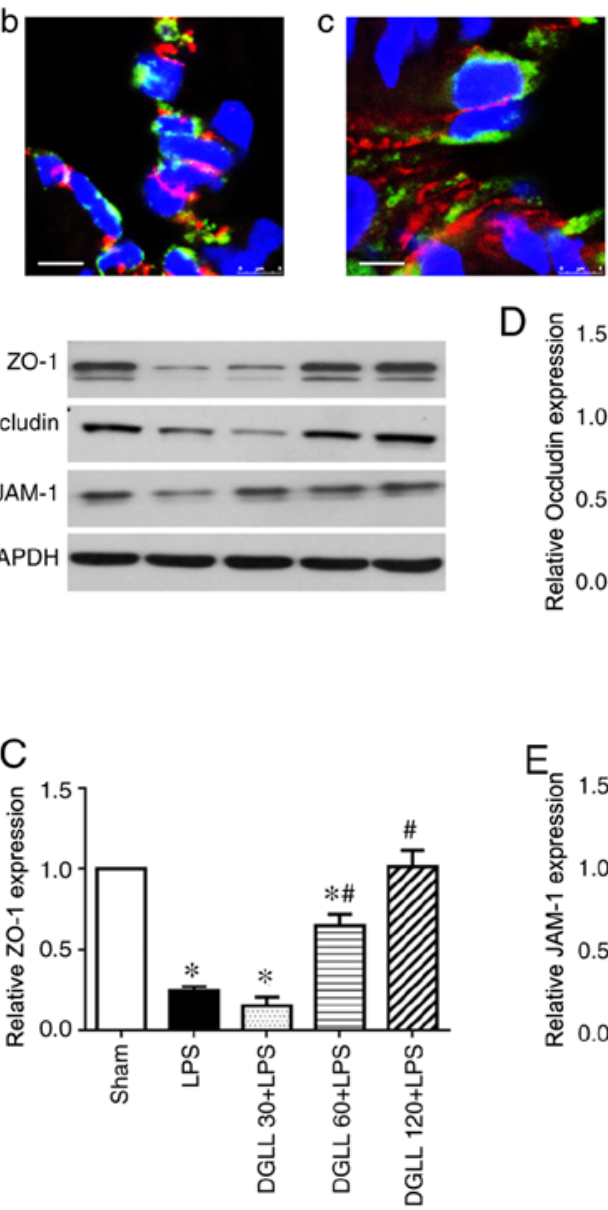

D
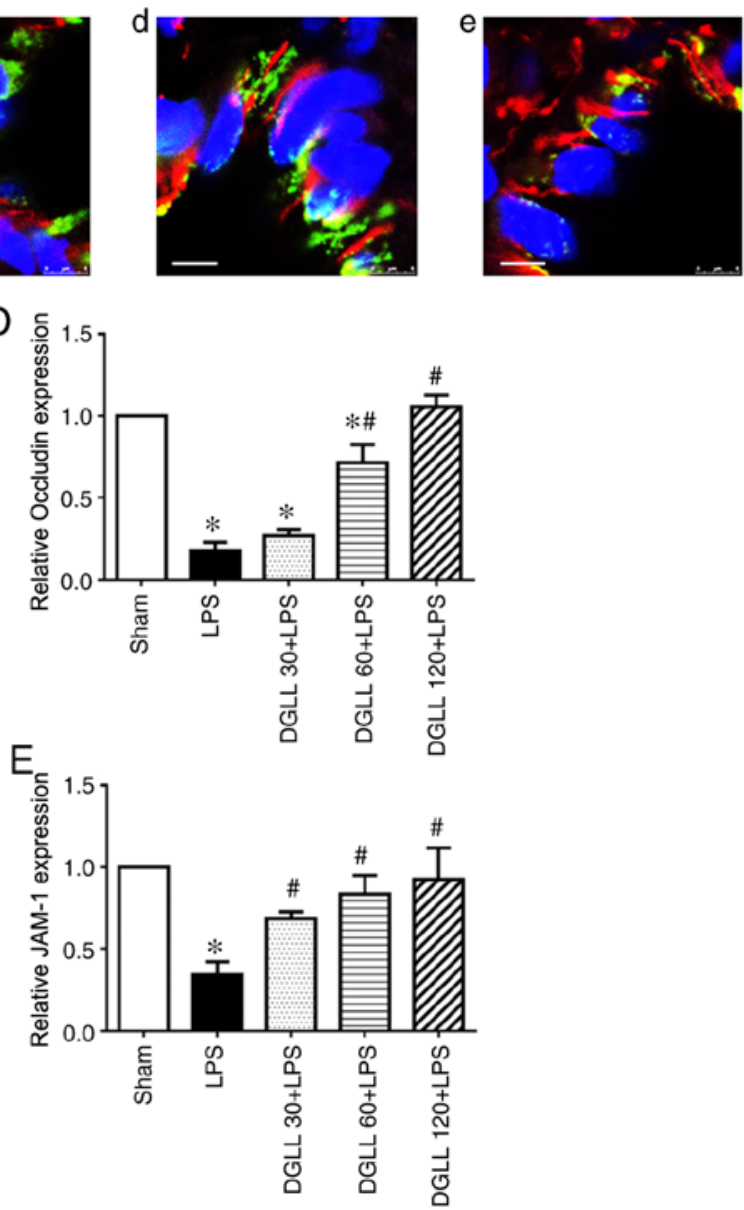

Figure 5. Effect of DGLL on the regulation of tight junction protein expression in rat lung tissue after LPS treatment. (A) Representative immunofluorescence confocal images for Occludin expression in lung tissues in (a) Sham, (b) LPS, (c) DGLL 30 + LPS, (d) DGLL 60 + LPS and (e) DGLL 120 + LPS groups. Red, Occludin; green, vWF; blue, Hoeschst 33342 (nuclear). Scale bar, $5 \mu \mathrm{m}$. (B) Representative western blot image and quantitative analysis of (C) ZO-1, (D) occludin and (E) JAM-1 in rat lung tissue. All data are expressed as the mean \pm SEM. ${ }^{*} \mathrm{P}<0.05$ vs. Sham; ${ }^{\#} \mathrm{P}<0.05$ vs. LPS. DGLL, diammonium glycyrrhizinate lipid ligand; LPS, lipopolysaccharide; JAM-1, junction adhesion molecule-1; ZO-1, zonula occludens-1; vWF, von Willebrand factor.

and JAM-1 in the rat lung tissue were significantly decreased following LPS induction (Fig. 5B-E). In the DGLL 60 and $120 \mathrm{mg} / \mathrm{kg}$ dose groups, lung tissue tight junction expression levels were significantly higher compared with those in the LPS group (Fig. 5B-E). This demonstrated that DGLL prevented the reductions in the expression of pulmonary tight junction proteins caused by LPS.

\section{Discussion}

The present study demonstrated that pretreatment with DGLL prevented LPS-induced ALI in rats, reduced MPO expression levels, reduced cytokine production and downregulated adhesion molecule expression in LPS-inflamed lung tissues. DGLL also attenuated LPS-induced pulmonary edema and pulmonary microvascular permeability in addition to preventing the reductions in the expression of the adhesion junction protein VE-Cadherin and tight junction proteins ZO-1, occludin and JAM-1 in rat lung tissues.

Recent studies have shown that damage to the pulmonary microvascular barrier and the resulting pulmonary edema is the primary pathological feature of early-stage ALI, serving as a key target for treating ALI/ARDS $(25,26)$. Epidemiological studies have previously revealed that LPS, which is a major component of the cell wall in gram-negative bacteria, is the most common cause of lung injury and has been widely used to establish animal models of ALI $(27,28)$. Previous studies have shown that LPS activates NF- $\mathrm{NB}$ by binding to leukocyte toll-like receptor- 4 receptors, inducing the expression of a number of inflammatory factors, including TNF- $\alpha$, IL-1 $\beta$ and IL-6, leading to the destruction of paracellular junctions (29,30). Consequently, by increasing the expression levels of adhesion molecules on the endothelium, LPS facilitates leukocyte adhesion to the microvascular endothelium (31). Adherent leukocytes indirectly damage the microvessels by releasing proteases and peroxides, which increases pulmonary microvascular permeability and lung edema, eventually resulting in decreased lung compliance and functional impairment (32). Previous pharmacological studies have documented that diammonium glycyrrhizinate decreases lung injury by decreasing inflammation in the respiratory tract, inhibiting protein and mRNA expression of inflammatory factors in the lung tissue and by suppressing the expression and activation of $N F-\kappa B(33,34)$. The present study verified further that DGLL inhibited LPS-induced inflammatory cell infiltration into the lung tissue and decreased the upregulation of MPO 
expression, which is a leukocyte infiltration marker (35). Additionally, DGLL also reduced the levels of inflammatory cytokines TNF- $\alpha$ and IL- $1 \beta$ and the expression of adhesion molecule ICAM-1 in lung tissues. In vitro data demonstrated that DGLL also inhibited LPS-induced cytokine release from RAW264.7 macrophages dose-dependently. These results suggest that DGLL inhibited the increase in pulmonary microvascular permeability by inhibiting the excessive activation of leukocytes in LPS-challenged lung tissues.

In addition to indirectly damaging blood vessels via leukocyte hyperactivation, LPS directly damages microvascular barrier function, which results in increased microvascular permeability and pulmonary edema $(36,37)$. Results of the present study demonstrated that the wet-to-dry weight ratio, BALF protein content and extravasation of EB in lung tissues in the DGLL pretreatment groups were significantly lower compared with those in the LPS group. These observations suggest that DGLL inhibited LPS-induced pulmonary microvascular hyperpermeability and pulmonary tissue edema. The microvascular barrier is primarily regulated by tight and adhesion junctions between microvascular endothelial cells (38). Adherens junctions are primarily formed by VE-cadherin protein via the formation of homodimers, which are linked to catenin proteins in the cytoplasm by cytoskeletal proteins, such as F-actin (39). Tight junction proteins, including claudin, occludin and JAM, serve key roles in stabilizing the tight interactions between cells by linking with the ZO family of proteins in the cytoplasm (38). The present study demonstrated that DGLL significantly prevented the LPS-induced phosphorylation of VE-cadherin in the rat lung tissues and LPS-induced reductions in VE-cadherin expression. A possible limitation of this study was that only the full-length of VE-cadherin was measured, where the analysis of VE-degradation fragment expression was missing. In addition, DGLL stabilized lung microvascular tight junction proteins occludin (Fig. 5A), as well as increasing the expression levels of JAM-1 and ZO-1 protein. DGLL regulated microvascular barriers in the lung tissue by increasing the expression levels of tight junction and adhesion proteins, thereby preventing LPS-induced pulmonary microvascular hyperpermeability and pulmonary tissue edema. However, the regulatory mechanism of DGLL on tight junction and adhesion proteins in microvascular endothelial cells, as well as the therapeutical effects of DGLL after ALI induction require further investigation.

In conclusion, DGLL exhibited significant protective effects against LPS-induced ALI in rats, with the mechanism of action of which found to be associated with the inhibition of inflammatory cell infiltration and reduced expression of intercellular junction proteins. These results provided a novel theoretical basis for the clinical treatment of ALI using DGLL.

\section{Acknowledgements}

Not applicable.

\section{Funding}

The present study was supported financially by the Key Projects of Natural Science Research of Anhui Province (grant no. KJ2016A374).

\section{Availability of data and material}

The datasets used and/or analyzed during the current study are available from the corresponding author on reasonable request.

\section{Authors' contributions}

MML performed and funded the research and analyzed the data. JZ and DJ contributed to animal experiments. JY and YPH performed the immunochemistry analysis. QW designed the research, interpreted the data, wrote the manuscript and finally approved the submission of this manuscript. All authors read and approved the manuscript.

\section{Ethics approval and consent to participate}

All animals were handled according to the guidelines of the Anhui Medical University Animal Research Committee. The protocols were approved by the Committee on the Ethics of Animal Experiments of the Anhui Medical University (approval nos. IACUC20180724-18 and IACUC20200710-11; Hefei, China).

\section{Patient consent for publication}

Not applicable.

\section{Competing interests}

The authors declare that they have no competing interests.

\section{References}

1. Piantadosi CA and Schwartz DA: The acute respiratory distress syndrome. Ann Intern Med 141: 460-470, 2004.

2. Rezoagli E, Fumagalli R and Bellani G: Definition and epidemiology of acute respiratory distress syndrome. Ann Transl Med 5: 282, 2017.

3. Bellani G, Laffey JG, Pham T, Fan E, Brochard L, Esteban A, Gattinoni L, van Haren F, Larsson A, McAuley DF, et al; LUNG SAFE Investigators; ESICM Trials Group: Epidemiology, patterns of care, and mortality for patients with acute respiratory distress syndrome in intensive care units in 50 countries. JAMA 315: 788-800, 2016.

4. Meduri GU, Golden E, Freire AX, Taylor E, Zaman M, Carson SJ, Gibson $M$ and Umberger R: Methylprednisolone infusion in early severe ARDS: Results of a randomized controlled trial. Chest 131: 954-963, 2007

5. Meduri GU, Headley AS, Golden E, Carson SJ, Umberger RA, Kelso T and Tolley EA: Effect of prolonged methylprednisolone therapy in unresolving acute respiratory distress syndrome: A randomized controlled trial. JAMA 280: 159-165, 1998.

6. Matthay MA, Ware LB and Zimmerman GA: The acute respiratory distress syndrome. J Clin Invest 122: 2731-2740, 2012.

7. Matthay MA,Zemans RL,Zimmerman GA, Arabi YM, Beitler JR, Mercat A, Herridge M, Randolph AG and Calfee CS: Acute respiratory distress syndrome. Nat Rev Dis Primers 5: 18, 2019.

8. Fan E, Brodie D and Slutsky AS: Acute respiratory distress syndrome: Advances in diagnosis and treatment. JAMA 319: 698-710, 2018.

9. Matthay MA and Zimmerman GA: Acute lung injury and the acute respiratory distress syndrome: Four decades of inquiry into pathogenesis and rational management. Am J Respir Cell Mol Biol 33: 319-327, 2005.

10. Cardinal-Fernández P, Bajwa EK, Dominguez-Calvo A, Menéndez JM, Papazian L and Thompson BT: The presence of diffuse alveolar damage on open lung biopsy is associated with mortality in patients with acute respiratory distress syndrome: A systematic review and meta-analysis. Chest 149: 1155-1164, 2016. 
11. Johnson ER and Matthay MA: Acute lung injury: Epidemiology, pathogenesis, and treatment. J Aerosol Med Pulm Drug Deliv 23: 243-252, 2010

12. Zhang R, Cheng K, Xu S, Li S, Zhou Y, Zhou S, Kong R, Li L, Li J, Feng J, et al: Metformin and diammonium glycyrrhizinate enteric-coated capsule versus metformin alone versus diammonium glycyrrhizinate enteric-coated capsule alone in patients with nonalcoholic fatty liver disease and type 2 diabetes mellitus. Gastroenterol Res Pract 2017: 8491742, 2017.

13. Pang H, Huang T, Song J, Li D, Zhao Y and Ma X: Inhibiting HMGB1 with glycyrrhizic acid protects brain injury after DAI via its anti-inflammatory effect. Mediators Inflamm 2016 $4569521,2016$.

14. Cai Y, Xu Y, Chan HF, Fang X, He C and Chen M: Glycyrrhetinic acid mediated drug delivery carriers for hepatocellular carcinoma therapy. Mol Pharm 13: 699-709, 2016.

15. He Y, Lou X, Jin Z, Yu L, Deng L and Wan H: Mahuang decoction mitigates airway inflammation and regulates IL-21/STAT3 signaling pathway in rat asthma model. J Ethnopharmacol 224: 373-380, 2018.

16. Zhong Y, Zhou J, Liang N, Liu B, Lu R, He Y, Liang C, Wu J, Zhou Y, Hu M, et al: Effect of maxing Shigan Tang on H1N1 influenza a virus-associated acute lung injury in mice. Intervirology 59: 267-274, 2016

17. Yao L and Sun T: Glycyrrhizin administration ameliorates Streptococcus aureus-induced acute lung injury. Int Immunopharmacol 70: 504-511, 2019.

18. Chen J, Zhang W, Zhang L, Zhang J, Chen X, Yang M, Chen T and Hong J: Glycyrrhetinic acid alleviates radiation-induced lung injury in mice. J Radiat Res (Tokyo) 58: 41-47, 2017.

19. Zhang D, Liu B, Cao B, Wei F, Yu X, Li GF, Chen H, Wei LQ and Wang PL: Synergistic protection of Schizandrin B and Glycyrrhizic acid against bleomycin-induced pulmonary fibrosis by inhibiting TGF- $\beta 1 / \mathrm{Smad} 2$ pathways and overexpression of NOX4. Int Immunopharmacol 48: 67-75, 2017.

20. Jung JC, Lee YH, Kim SH, Kim KJ, Kim KM, Oh S and Jung YS: Hepatoprotective effect of licorice, the root of Glycyrrhiza uralensis Fischer, in alcohol-induced fatty liver disease. BMC Complement Altern Med 16: 19, 2016.

21. Tsuruoka N, Abe K, Wake K, Takata M, Hatta A, Sato T and Inoue $\mathrm{H}$ : Hepatic protection by glycyrrhizin and inhibition of iNOS expression in concanavalin A-induced liver injury in mice. Inflamm Res 58: 593-599, 2009.

22. Kan C, Ding N, Yang J, Tan Z, McGuire TL, Lu H, Zhang K, Berger DM, Kessler JA and Kan L: BMP-dependent, injury-induced stem cell niche as a mechanism of heterotopic ossification. Stem Cell Res Ther 10: 14, 2019.

23. Matute-Bello G, Downey G, Moore BB, Groshong SD, Matthay MA, Slutsky AS and Kuebler WM; Acute Lung Injury in Animals Study Group: An official American Thoracic Society workshop report: Features and measurements of experimental acute lung injury in animals. Am J Respir Cell Mol Biol 44: 725-738, 2011.

24. Chan YH, Harith HH, Israf DA and Tham CL: Differential regulation of LPS-mediated VE-cadherin disruption in human endothelial cells and the underlying signaling pathways: A mini review. Front Cell Dev Biol 7: 280, 2020.

25. Dong W, He B, Qian H, Liu Q, Wang D, Li J, Wei Z, Wang Z, $\mathrm{Xu} \mathrm{Z}, \mathrm{Wu} \mathrm{G}$, et al: RAB26-dependent autophagy protects adherens junctional integrity in acute lung injury. Autophagy 14: $1677-1692,2018$
26. Audard J, Godet T, Blondonnet R, Joffredo JB, Paquette B, Belville C, Lavergne M, Gross C, Pasteur J, Bouvier D, et al: Inhibition of the receptor for advanced glycation end-products in acute respiratory distress syndrome: A randomised laboratory trial in piglets. Sci Rep 9: 9227, 2019.

27. Chen H, Bai C and Wang X: The value of the lipopolysaccharide-induced acute lung injury model in respiratory medicine. Expert Rev Respir Med 4: 773-783, 2010.

28. Cheng KT, Xiong S, Ye Z, Hong Z, Di A, Tsang KM, Gao X, An S, Mittal M, Vogel SM, et al: Caspase-11-mediated endothelial pyroptosis underlies endotoxemia-induced lung injury. J Clin Invest 127: 4124-4135, 2017.

29. Tong W, Chen X, Song X, Chen Y, Jia R, Zou Y, Li L, Yin L, He C, Liang X, et al: Resveratrol inhibits LPS-induced inflammation through suppressing the signaling cascades of TLR4-NF-кB/MAPKs/IRF3. Exp Ther Med 19: 1824-1834, 2020.

30. Lee YM, Hybertson BM, Cho HG, Terada LS, Cho O, Repine AJ and Repine JE: Platelet-activating factor contributes to acute lung leak in rats given interleukin-1 intratracheally. Am J Physiol Lung Cell Mol Physiol 279: L75-L80, 2000.

31. Zemans RL, Colgan SP and Downey GP: Transepithelial migration of neutrophils: Mechanisms and implications for acute lung injury. Am J Respir Cell Mol Biol 40: 519-535, 2009.

32. Abraham E: Neutrophils and acute lung injury. Crit Care Med 31 (Suppl 4): S195-S199, 2003

33. Feng $\mathrm{C}$, Wang $\mathrm{H}$, Yao $\mathrm{C}$, Zhang $\mathrm{J}$ and Tian Z: Diammonium glycyrrhizinate, a component of traditional Chinese medicine Gan-Cao, prevents murine T-cell-mediated fulminant hepatitis in IL-10- and IL-6-dependent manners. Int Immunopharmacol 7 : 1292-1298, 2007.

34. Jin J, Xiong T, Hou X, Sun X, Liao J, Huang Z, Huang M and Zhao Z: Role of Nrf2 activation and NF- $\kappa$ B inhibition in valproic acid induced hepatotoxicity and in diammonium glycyrrhizinate induced protection in mice. Food Chem Toxicol 73: 95-104, 2014.

35. Ding D, Xu S, Zhang H, Zhao W, Zhang X, Jiang Y, Wang P, Dai Z and Zhang J: 3-Methyladenine and dexmedetomidine reverse lipopolysaccharide-induced acute lung injury through the inhibition of inflammation and autophagy. Exp Ther Med 15: 3516-3522, 2018

36. Dreymueller D, Martin C, Kogel T, Pruessmeyer J, Hess FM, Horiuchi K, Uhlig S and Ludwig A: Lung endothelial ADAM17 regulates the acute inflammatory response to lipopolysaccharide. EMBO Mol Med 4: 412-423, 2012.

37. Veszelka S, Pásztói M, Farkas AE, Krizbai I, Ngo TK, Niwa M, Abrahám CS and Deli MA: Pentosan polysulfate protects brain endothelial cells against bacterial lipopolysaccharide-induced damages. Neurochem Int 50: 219-228, 2007.

38. Mehta D and Malik AB: Signaling mechanisms regulating endothelial permeability. Physiol Rev 86: 279-367, 2006.

39. Rimm DL, Koslov ER, Kebriaei P, Cianci CD and Morrow JS: Alpha $1(\mathrm{E})$-catenin is an actin-binding and -bundling protein mediating the attachment of F-actin to the membrane adhesion complex. Proc Natl Acad Sci USA 92: 8813-8817, 1995.

This work is licensed under a Creative Commons Attribution-NonCommercial-NoDerivatives 4.0 International (CC BY-NC-ND 4.0) License. 